\title{
La catalogación del arte prehistórico: el «corpus» de pinturas rupestres de Cataluña
}

\author{
Eduardo Ripoll Perello
}

La fase de inventario es previa a cualquier otro trabajo en el campo del patrimonio histórico y artístico, y naturalmente en el del arte prehistórico. El de este último no ha tenido hasta ahora mucha suerte. $Y$, sin embargo, inventariar y sistematizar las fuentes -iconográficas, escritas, epigráficas, de la cultura material, etc.- es elevarlas a la condición superior de documentos.

En la historiografía del arte prehistórico se cuenta con diversos intentos de establecer unos corpora, cuya utilidad general estuvo y está fuera de duda. Aunque no se han conseguido series exhaustivas, hay que consolarse con el hecho cierto de la importancia de la literatura al respecto que se ha ido produciendo en menos de un siglo.

Desde los comienzos del estudio del arte prehistórico, sus investigadores sintieron la necesidad de una exposición metódica que diera unidad a sus publicaciones y que estas constituyeran catálogos o formaran series. Los ejemplos más antiguos de inventario sistemático son el pequeño libro de Salomon Reinach y la gran obra póstuma de Edouard Piette dedicada al arte mueble paleolítico francés ${ }^{1}$. Luego, en aquel sentido de seriación se ordenaron las grandes monografías editadas gracias a la munificiencia del Príncipe Alberto I de Mónaco, con las que se quería

' Salomon REINACH, Répertoire de l'art quaternaire, Paris, Leroux, 1913, 205 págs. y 189 figs.-Edouard PIETTE, L'art pendant l'Age du Renne, París, Masson, 1907, 112 págs., 126 figs. y 100 láminas. 
constituir una colección sistemática y completa. Pero, desgracidamente, no tuvieron continuidad a causa de las crisis consecutivas a la Primera Guerra Mundial y a la muerte de aquel gran mecenas, que tanto afectaron a su "Institut de Paléontologie Humaine» de Paris. En efecto, a la primera monografía de Altamira de 1906, para España pronto le hicieron compañia Les cavernes de la région cantabrique, el enorme volumen publicado por H. Alcalde del Rio, H. Breuil y L. Sierra en 1913; el mismo año se editó La Pasiega à Puente Viesgo, obra de $\mathrm{H}$. Breuil, $\mathrm{H}$. Obermaier y $\mathrm{H}$. Alcalde del Rio, cueva que no habia tenido cabida en la dedicada a las cavernas cantábricas; y dos años después, en 1915, $\mathrm{H}$. Breuil, $H$. Obermaier y Willoughby Verner publicaban La Pileta à Benaojan ${ }^{2}$. Mientras tanto, para el arte paleolítico francés, en 1910, L. Capitan, $H$. Breuil y Denis Peyrony habian publicado La caverne de Font de Gaume aux Eyzies, y hasta 1924 no aparecería Les Combarelles aux Eyzies de los mismos autores ${ }^{3}$.

Estaba en la mente del Abate Breuil y en la de sus colaboradores que debian publicarse otros trabajos de dicho tipo para otras cuevas con menor cantidad de representaciones artisticas y por regiones geográficas. En este sentido se trabajó en la preparación de una gran monografía dedicada al arte de las cavernas del Pirineo francés semejante a la consagrada a sus hermanas cantábricas. Pero, la situación económica posterior a la guerra de 1914-1918, a la que ya hemos aludido, obligaron a renunciar al proyecto. La serie patrocinada por el Principe de Mónaco, a pesar de los muchos años transcurridos desde su publicación, sigue siendo el más ambicioso proyecto de publicación científica y el realizado con mejor presentación hasta ahora entre las ediciones dedicadas al arte prehistórico.

Emile CARTAll hac, y Henri Breuil, La caverne d'Altamira à Santillane, près Santander (Espagne), Mónaco, 1906, 287 págs. 205 figs. y 38 láminas.-Hermilio Alcalde del Rio, Henri Breul, y Lorenzo Sierra, Les cavernes de la région cantabrique (Espagne), Mónaco, Chêne, 1912, 265 págs., 258 figs. y 100 láminas.-Henri Breull. Hugo Obermaier, y Hermilio Alcalde oel Rio, la Pasiega à Puente Viesgo (Santander); Mónaco, Chêne, 1913.-Henri Breull; Hugo Obermaier, y Willoughby Verner, La Pileta à Benaojan (Málaga), Mónaco, Chêne, 1915, 68 págs., 26 figs. y 22 láminas.-Años después, la segunda gran monografia sobre Altamira enlazó con esta colección: Henri Breull, y Hugo Obermaier, La cueva de Altamira en Santillana del Mar, Madrid, Academia de la Historia, 1935, 236 págs., 183 figs. y 52 láminas (también se hizo una versión en inglés)

3 Louis Capitan. Henri Breull, y Denis Peyrony, La caverne de Font-de-Gaume aux Eyzies (Dordogne). Mónaco, Chêne, 1910, 217 págs., 244 figs. y 65 láminas.-CAPITAN, Breuil y Peyrony, Les Combarelles aux Eyzies (Dordogne), París, Masson, 1924, 192 págs., 128 figs. y 63 láminas. 
Independientemente de su colaboración con los franceses, también los estudiosos españoles sintieron aquella necesidad. Recordemos que el primer libro de conjunto sobre el arte postpaleolítico español, obra de Juan Cabré y Aguiló, ya tenía la pretensión de un corpus ${ }^{4}$. El Arte Rupestre en España (1915) fue el primero de los volúmenes de la bella serie de Memorias de la madrileña "Comisión de Investigaciones Paleontológicas y Prehistóricas" que fundó y dirigió el prof. Eduardo HernándezPacheco. Pero la obra de Cabré se editaba por pliegos y prontó el investigador bajoaragonés se dio cuenta de que excedería las dimensiones de un volumen normal. Por ello la parte fundamental de su obra la dedicó al arte levantino y en buena parte al esquemático. Para su tiempo y para estas dos provincias artísticas, el libro cumplió aquella función.

Además del libro de Cabré, en dicha colección de memorias hay seis tomos dedicados al arte prehistórico, debidos a Cabré. Hernández-Pacheco, Obermaier, el Conde de la Vega del Sella y Paul Wernert. Especialmente notable fue la monografía dedicada por Hernández-Pacheco a la Cueva de la Araña, en la que, como complemento, estableció por primera vez una cronología postpaleolítica para el arte levantino frente a las teorías en boga del Abate Breuil ${ }^{5}$. En este caso, a causa de los acontecimientos políticos de los años veinte y treinta, la serie también quedó truncada. Pero Hernández-Pacheco no perdió la esperanza de que se podria llegar al deseado corpus y por ello siguió en aquella época con su labor de campo. Para su trabajo contaba con las extraordinarias facultades del dibujante Francisco Benítez Mellado. Por suerte se conservan muchos de los calcos de este equipo tanto en el Museo Nacional de Ciencias Naturales como en el Museo Arqueológico Nacional, ambos en Madrid.

4 Juan CABre y AGuilo, El arte rupestre en España. Región septentrional y oriental, Memorias de la Comisión de Investigaciones Paleontológicas y Prehistóricas, núm. 1. Madrid. 1915.

- Eduardo Hernandez-Pacheco, Las pinturas prehistóricas de Peña Tu (memoria núm. 2, 1914).-Juan CABRE, y Eduardo Hernanuez-PACheCo, Avance.al estudio de las pinturas prehistoricas del extremo sur de España (Laguna de la Janda) (memoria núm. 3, 1914).-Juan CABRÉ y Aguilo, Las pinturas rupestres de Aldeaquemada (memoria núm. 14. 1917). - Eduardo Hernandez-PACHECo, Los grabados de la cueva de Penches (memoria núm. 17, 1917). - Hugo Obermaier, y Conde de la Vega del Sella, la cueva del Buxu (Asturias), (memoria núm. 20, 1918).-Hugo Obermaier, Paul Wernert, Las pinturas rupestres del Barranco de Valltorta (Castellón) (memoria núm. 23 (1919).-Eduardo HeRnandezPACHECo, La caverna de la Peña del Candamo (Asturias), (memoria num. 24, 1919). - Eduardo HeRnandez-PACHECo, Las pinturas prehistoricas de la Araña (Valencia). Evolución del arte rupestre de España (memoria nüm. 34, 1924) 
Un paso adelante en la idea de que nos estamos ocupando lo constituyó sin duda, en los años treinta, y referida al llamado arte esquemático, la monumental obra del Abate Henri Breuil, Les peintures schématiques de la Péninsule lbérique ${ }^{6}$, publicada por la "Fondation Singer-Polignac", de París, que constituye en realidad el único verdadero corpus que poseemos hasta ahora para una provincia de arte prehistórico del territorio peninsular. Se trata de una obra todavía fundamental aunque notemos en ella el paso de los años, con el correspondiente mejoramiento de las técnicas, y el hecho de ser un trabajo casi individual del inolvidable maestro. El propio Abate Breuil, en los años cincuenta, nos hablaba de la necesidad de un volumen suplementario de su obra que recogiera lo descubierto en los dos decenios transcurridos desde su publicación.

Después de la guerra civil española, el profesor Martín Almagro Basch, desde Barcelona y con el concurso de Francisco Benitez Mellado, conforme a la planificación que había hecho Hernández-Pacheco, volvió a la idea de publicaciones basadas en calcos. El proyecto decayó en los años cincuenta con la marcha a Chile de Benítez Mellado. Hicimos entonces los calcos de todos los abrigos de Alacón y de algunos de Albarracín. Luego, Almagro, ya en Madrid, siguió pensando en el proyecto. Para él, el malogrado fotógrafo Gil Carles realizó gran número de fotografías en los años sesenta. En este momento, el auge de la técnica del color hacia pensar que el corpus podía ser hecho a base de fotografías.

Nosotros mismos, desde Barcelona, teniamos conciencia del problema y de él habiamos discutido muchas veces con el profesor Luis Pericot. Fue el propio Abate Breuil quien saludó con alegría nuestro propósito de publicar la colección Monografias de Arte Rupestre, en la que deseaba fueran incluidas sus propias investigaciones francesas y españolas de principios de siglo dispersas en numerosas revistas (Gargas, Niaux, Alpera, Minateda, etc.). Esta serie pudo iniciarse y empezar su andadura gracias a la ayuda de la "Wenner-Gren Foundation for Anthropological Research", de Nueva York, que apoyó los trabajos de investigación y sufragó la edición en inglés de las obras publicadas referentes a la Península Ibérica. En nuestro país, contrariamente a lo que se esperaba, la única ayuda recibida lo fue de la Diputación de Barcelona que hizo suya la versión castellana de la serie a través de su «Instituto de Prehistoria y Arqueología". De esta colección se llegaron a publicar las monografias

- Henri BREUIL, Les peintures rupestres schématiques de la Péninsule lbérique, IV vols., Lagny, Fondation Singer-Polignac, 1933-1935.-Su sistema de corpus de las pinturas esquemáticas ya estaba preanunciado en el libro de Henri BreuIt, y Miles BuRKIT, Rock paintings of Southern Andalusia, Oxford, 1929. 
de arte levantino de Santolea y del Cingle de La Gasulla ${ }^{7}$, las de Las Monedas y las Chimeneas para el arte paleolítico ${ }^{8}$, e incluso se intentó extender el proyecto al otro lado del Atlántico con la edición en un volumen de algunos estudios del arte rupestre de Argentina ${ }^{9}$. Pero, a pesar de las generosas contribuciones indicadas, el empeño era demasiado ambicioso y rebasaba las posibilidades económicas y humanas con que podiamos contar. Quedaron por publicar, por ejemplo, el catálogo de las representaciones rupestres de la cueva del Castillo (Cantabria) o el arte rupestre de Masmas (Nubia egipcia), por señalar sólo trabajos personales del que esto escribe.

Para tiempos más próximos debemos citar el esfuerzo realizado por A. García Alén y A. de la Peña Santos en la catalogación de los petroglifos gallegos de la provincia de Pontevedra ${ }^{10}$. Se trata de una región artistica de gran singularidad y muy necesitada de un corpus total pues su documentación es de las más dispersas.

Por último, debemos citar la iniciativa del profesor Francisco Jordá Cerdá para publicar un Corpus Artis Rupestris, con varias secciones y en un formato muy acertado. Del mismo, por lo que sabemos, sólo han aparecido dos fascículos dedicados a la cueva Navarro (Málaga) y la cueva de La Griega (Segovia), debidos a J. L. Sanchidrián y Georges y Suzanne Sauvet respectivamente, y ambos en la sección Palaeolitica Ars ${ }^{11}$.

7 Eduardo Ripoll Perello, Los abrigos pintados de los alrededores de Santolea (Teruel), Barcelona, 1961 (en inglés, 1967), nota preliminar del Abate Henri Breuil, prólogo de Luis Pericot, Barcelona, Instituto de Prehistoria y Arqueologia y Wenner Gren Foundation, 36 págs., 8 figs. y VIII láminas; Id., Pinturas rupestres de la Gasulla (Castellón), con reproducción de calcos del Abate $\mathrm{H}$. Breuil, Barcelona, Instituto de Prehistoria y Arqueología y Wenner Gren Foundation, 1963 (en inglés, 1968), 60 págs., 34 figs. y XXXV láminas.

${ }^{8}$ Eduardo Ripoll Perello, La cueva de Las Monedas en Puente Viesgo (Santander), Barcelona, Instituto de Prehistoria y Arqueologia y Wenner Gren Foundation, 1972 (en inglés, 1980), 67 págs., 30 figs. y XXXV láminas. Joaquín Gonzalez EcheGaray, Pinturas y grabados de la Cueva de Las Chimeneas (Puente Viesgo, Santander), Barcelona, Instituto de Prehistoria y Arqueologia y Wenner Gren Foundation, 1974, 44 págs., 22 figs. y XXIII láminas

${ }^{9}$ Lidia C. Alfaro lanzone, Nicolás de la Fuente, Adán Roberto Diaz Romero, Mily S. de Ragigio, Jorge Fernandez, y Carlos J. Gradin, Miscelánea de arte rupestre de la República Argentina, Introducción por Juan Schobinger y E. Ripoll-Perello, Barcelona, Instituto de Prehistoria y Arqueología y Wenner Gren Foundation, 1979, 174 págs. con figs. y láminas.

10 A. Garcia Alen, y A. de Pena Santos, Grabados rupestres de la provincia de Pontevedra, Pontevedra-La Coruña, Fundación Pedro Barrie de la Maza y Museo de Pontevedra, 1980, 229 págs., 166 figs. y 131 fotografias.

" José Luis, Sanchidrian Torti, Cueva Navarro (Cala del Moral, Málaga), prólogo de Francisco Jorda Cerdá. Salamanca, 1981, 30 págs., 43 figs. y VIII láminas. Georges y 
$Y$, aunque de él no se ha publicado nada, señalaremos los trabajos para la confección de un corpus patrocinado por el Ministerio de Cultura y su Dirección General de Bellas Artes, que debía corresponder a la actualización de la idea con más medios y en cuya planificación intervinimos en sus comienzos. Se trataba de ofrecer al estudioso una ficha general de cada yacimiento con indicación de sus caracteristicas, número y tipo de obras de arte que contiene, bibliografía, planos y fotografías. Se recopilaron numerosas fichas redactadas por diversos autores. El inventario fotográfico es una parte esencial de este proyecto. Del mismo, según nuestras informaciones, el equipo formado por Sergio Ripoll López, Fernando Piñón Varela (†) y José Latova, con algunos colaboradores, tiene terminado el inventario fotográfico de las obras de arte de las cuevas de la región cantábrica. Pero también este intento parece haber quedado parado por falta, otra vez, de los necesarios apoyos económicos de las autoridades competentes.

Como se puede ver se está lejos de conseguir en España un inventario del arte prehistórico. Como en tantas cosas de la investigación humanistica, hay que considerar esto como un fracaso. En el camino andado se ha perdido un tiempo precioso. En cambio, en Francia, hubo conciencia de aquella necesidad y desde 1984 los estudiosos franceses cuentan con un excelente y amplio corpus del rico arte paleolítico parietal de su territorio. Nos estamos refiriendo a L'Art des cavernes que, por su calidad, enlaza con las espléndidas publicaciones francesas a principios de siglo ${ }^{12}$.

Como hemos visto con la historia hasta aqui narrada, las dificultades para llegar a conseguir la edición de colecciones sistemáticas de arte prehistórico han sido sobre todo de tipo económico y administrativo en unos tiempos de gobierno excesivamente centralizado. Ahora, desde hace algunos años, se ofrecen a los investigadores españoles unas nuevas posibilidades. En efecto, seguramente la solución para este problema sería que se hicieran cargo de la catalogación del arte prehistórico las Comunidades Autónomas que tienen traspasados los servicios de Arqueología. Al parecer algunas de ellas han puesto en marcha los previos programas de documentación. A su cabeza se ha situado la Generalidad

Suzanne Sauvet, Los grabados rupestres prehistóricos de la cueva de La Griega (Pedraza. Segovia). Salamanca, 1983, 30 págs., 20 figs. y 28 fotografías.

AA. VV., L'Art des cavernes. Atlas des grottes ornées paleolithiques françaises, prólogo de André Leroi-Gourhan, París, Ministére de la Culture et Imprimerie Nationale, 1984. 676 págs. con figs. y planos. 
de Cataluña con la publicación del primer volumen del corpus de pinturas rupestres en territorio catalán, al que nos referiremos a continuación ${ }^{13}$.

Dicha publicación está dedicada a los abrigos pintados de la cuenca del rio Segre, tan importante como paso natural entre el Ebro y el Pirineo. Se nos presenta bajo la forma de un album que contiene un fasciculo de "generalidades" y otros trece que corresponden a otros tantos conjuntos con pinturas (con la posibilidad de añadir en el mismo album otros fasciculos a que den lugar futuros y muy previsibles hallazgos). Lo ahora publicado es el resultado del trabajo sobre el terreno de varios especialistas, en particular Anna Alonso Tejada para la documentación y la fotografía y Ramón Viñas Vallverdú para los planteamientos de base y en concreto para las planimetrías.

Como hemos dicho, en su estado actual, esta primera entrega del corpus catalán se refiere a pequeños conjuntos pictóricos de aquella zona geográfica, que son los siguientes (con indicación, entre paréntesis, del municipio en que se encuentran):

1. Abric de la Vall d'Ingla (Bellver de Cerdanya).

2. Roc del Rumbau (Peramola).

3. Cova del Cogulló (Vilanova de Meià).

4. Les Aparets, I, II y III (Alós de Balaguer).

5. Antona I, II y III (Artesa de Segre).

6. Balma del Pantà (Camarasa).

7. Cova del Tabac (Camarasa).

8. Cova dels Vilassos o dels Vilars (Os de Balaguer).

9. Alfés (Alfés).

10. Roques Guàrdies (les Borges Blanques).

11. Vall de la Coma (l'Albi).

12. Barranc de Sant Jaume (la Granja d'Escarp).

13. Barranc de Canà o de la Mina Federica (la Granja d'Escarp).

A los efectos de una apreciación global, de dicha lista habría que excluir en núm. 6 (con una informe mancha de color) y el núm. 10 (con una decena de pequeños trazos sin coherencia aparente). Para cada

(Josep Castells i CAMP, dir.), Inventari del Patrimoni Arqueologic de Catalunya. Corpus de pintures rupestres. I. La conca del Segre. Barcelona. Diputacio de Lleida i Direcció General del Patrimoni Cultural de la Generalitat de Catalunya, 1990. 14 fasc. de 8/10 págs. c. u., con ilustraciones, todo en un álbum $(32 \times 25 \mathrm{~cm})$. La obra va acompañada de un vídeo. 
lugar se indican, de forma sintética, las siguientes características: antecedentes, situación geográfica, descripción de las pinturas (situación sobre la roca, medidas, técnica, color - Pantone-, estilo, etc.), estado de conservación, observaciones cronológicas, documentación, topografía, calcos y fotografía.

En dicha estimación de conjunto habrá que tener en cuenta, además, que en la lista transcrita falta el importante conjunto de Cogul. Con muy buen acuerdo, por su importancia en todos los aspectos, en particular por la cantidad de sus figuras pintadas y grabadas y para el tema de la sucesión estilistica, en la obra que motiva estas líneas se anuncia que se consagrará una monografía especial al friso de Cogul. Como es bien sabido, Cogul fue objeto de un excelente libro de Martín Almagro Basch en $1952^{14}$. No creemos que desde entonces se haya podido adelantar mucho en la lectura de sus pinturas, aunque es seguro que existen más grabados. Por otra parte es posible que el conjunto haya perdido algo, haciéndose más dificil su siempre escasa visibilidad ${ }^{15}$. En lo que indudablemente se podrá decir algo más es en lo referente a los problemas de la cronología relativa, especialmente en relación con los descubrimientos del resto de la Peninsula en los últimos años. Probablemente no ocurrirá lo mismo con la cronología absoluta, ya que en su obra el profesor Almagro puso particular atención al tratamiento amplio de su problemática, reuniendo todos los datos en favor de la cronología postpaleolítica que él mismo había manejado en muchos trabajos anteriores contra las teorías del Abate Breuil y que eran continuación de los iniciados por Hernández-Pacheco tres decenios antes ${ }^{16}$. Para las fechas absolutas pensamos que tan sólo habrá de sumar lo mucho que se ha avanzado

\footnotetext{
${ }^{14}$ Martin Almagro Basch, El covacho con pinturas rupestres de Cogul (Lerida). Lérida, Instituto de Estudios lierdenses, 1952.

is En 1982 un desalmado desconocido arrojo el aceite de una lata de sardinas sobre las pinturas a través de la reja protectora. A los pocos dias pudimos comprobar personalmente el desaguisado. Por suerte, el polvo llegado de la vecina carretera. que estaba en obras, sirvió de absorbente de la grasa y en una nueva visita al cabo de un año no vimos huellas del estropicio. Probablemente en ello influyo el que las pinturas de Cogul esten tan embebidas en la roca. - Diversas modificaciones y nuevos grabados pueden verse en el calco de Ramón VINAS, Anna ALONSO y Elisa SARRIA. "Noves dades sobre el conjunt rupestre de la Roca dels Moros (Cogul, les Garrigues, Lleida)", Tribuna d'Arqueologia (Barcelona), 1986-1987, págs. 31-39, 2 figuras. Suponemos que dicho calco, con el detalle suficiente, servirá como base a la nueva monografia.

${ }^{16}$ El Abate Breuil leyó su último trabajo sobre el arte levantino en el simposio de Wartenstein en 1960, pero no pudo ser incluido en el libro que contiene los trabajos allí presentados. Lo hemos editado en: Henri BREUIL, «Les roches peintes leptolithiques de l'Espagne orientale", Ars Praehistorica, V-VI, 1986-1987, págs. 21-30 (precedido de E. RI. poll Perello, "A los veinticinco años de la muerte del Abate Henri Breuil", págs. 13-19).
} 
en tiempos recientes en el conocimiento de las diversas facies del Neolítico, Calcolítico y Edad del Bronce. Para la cronología relativa, señalaremos nuestros propios trabajos personales, que en absoluto consideramos definitivos ${ }^{17}$.

Celebramos, como es de justicia, la tan digna y bien presentada publicación del primer tomo del corpus de arte prehistórico en Cataluña, y hacemos cordiales votos por su continuidad. También los hacemos para que pronto se inicien otros semejantes que vayan recopilando los tesoros de arte rupestre prehistórico que se hallan por casi todas las comarcas de la geografía peninsular. La Generalidad de Cataluña se ha puesto ahora en cabeza de esta tarea al iniciar la publicación sistemática de los conjuntos que se encuentran en su territorio. A partir de ahora, para cualquier estudio sobre el arte esquemático, será obligado recurrir a este álbum y a los que sin duda pronto le seguirán.

Pero, para ir teminando esta nota, seános permitido hacer alguna observación derivada del hecho de que el autor de la misma hace más de cuarenta años que trabaja en esta temática y en el espacio geográfico levantino que incluye por el norte el valle del Segre ${ }^{18}$.

En primer lugar nos preguntamos - aunque el titulo ya avisa que se trata de pinturas rupestres- porque no se han publicado al mismo tiempo los grabados rupestres de la zona, como, que ahora recordemos, los de Peramola, Mequinenza, Maials (La Socarrada), Guimerà (Vall de Pous), o del Mas de N'Olives de Torreblanca (y aquí es obligado mencionar el nombre de nuestro viejo amigo Lluís Díez-Coronel i Montull, a cuyo

Entre otros en los siguientes: Eduardo RIPOLL PERELLO, «Para una cronologia relativa de las pinturas rupestres del Levante español", (G. FrEUnd, ed.), Festschrift für Lothar Zotz, Steinzeitfrage der Alten und Neuen Welt, Bonn, 1960, págs. 457-465; ID., «Para una cronologia relativa del arte levantino español», (Luis PERICOT Garcia, y Eduardo, RIPOLL PERELLO, eds.), Prehistoric Art of the Western Mediterranean and the Sahara, Nueva York, 1968 (Viking Fund Publications, núm. 39), págs. 167-175; ID., "Cuestiones en torno a la cronología del arte postpaleolítico en la Península Ibérica", (E. RIPOLL, ed.), Simposio internacional de Arte Rupestre, Barcelona, 1966, Barcelona, Instituto de Prehistoria y Arqueología, 1968, págs. 165-192. Y recientemente en: "Acerca de algunos problemas del arte postpaleolitico en la Peninsula Iberica", ETF, serie I (Prehistoria y Arquelogia), t. 3, 1990, págs. 71-104.

* En los años cincuencia y sesenta empleamos varias campañas a la búsqueda de aite rupestres paleolitico en algunas zonas pirenaicas de la Cerdanya y altos valles de los rios Segre y Noguera Pallaresa, pero sin resultados. Incluso en las epocas de maximo frio de Wurm, los artistas que pintaron y grabaron las grandes cavernas de la vertiente francesa, pudieron pasar en verano los collados pirenaicos hacia tierras meridionales. Los descubrimientos del rio Vero (Huesca), a los que nos referimos a continuación, lo han demostrado. 
apasionado interés se deben tantos descubrimientos de arte rupestre en la cuenca del Segre y otras comarcas leridanas) ${ }^{19}$. En la obra que estamos comentando, sólo en dos casos se describen un grabado radial y otro bitriangular - al parecer este superpuesto a la pintura-, ambos en el Barranc de Sant Jaume. ¿Se prescindirá del contexto grabado cuando se publique el conjunto de Cogul? Seria de lamentar y haría su estudio incompleto. ¿Y los cruciformes de las proximidades del mismo Cogul que dieron a conocer Pere Bosch Gimpera y Josep Colominas? ${ }^{20}$

Acertadamente también, en este caso por ser un dato negativo, se ha prescindido de las pretendidas pinturas de la Cova Colomera o de Les Gralles (Sant Esteve de la Sarga, Pallars Jussà), para las que se dictaminó que se trataba de simples manchas de color de descomposición de la piedra soporte.

Otra observación: los paralelos. En estos estudios, la comparación iconográfica es obligada para las zonas geograficamente inmediatas, haciéndose menos precisa cuando se hace con regiones alejadas. Con ello se pueden precisar y justificar las atribuciones cronológicas. Por ejemplo, dentro del mismo valle del Segre, y por tanto en esta misma publicación, el cuadrúpedo del Barranc de Caná tiene su más cercano paralelo en Cogul (escena de la parte superior izquierda). Y, aunque no se hace en el corpus que motiva este escrito, creemos que las pinturas del valle del Segre debían haber sido puestas en relación con las de los sitios oscenses, cuya distancia a vuelo de pájaro no es superior a los $80 \mathrm{~km}$. Nos referimos a las pinturas esquemáticas de Lecina publicadas por Antonio Beltrán y a la treintena de lugares con arte paleolitico y de las facies levantina y esquemática del postpaleolítico que se hallan en la barrancadas de la cuenca alta del río Vero (Cueva de la Fuente del Trucho, Labarta, Arpán, Chimiachas, etc.) descubiertos y estudiados de manera modélica por Vicente Baldellou y sus colaboradores ${ }^{2 i}$. Esta concentración de diversos estilos en una región tan pequeña como es la del rio

L. Diez-Coronel Montull, "Los grabados rupestres prehistóricos de Mas de N'Olives, en Torreblanca (Lérida)", Ilerda, XLIII, 1982, pags. 17-39, 7 figs. y 22 fotografias. ID., "La roca con grabados del Mas de N'Olives en Torreblanca (Lérida)", Ars Praehistorica, VVI, 1986-1987, págs. 71-101, 32 figuras.

20 P. Bosch Gimpera, y J. Colominas Roca, "Pintures i gravats rupestres", Anuari de I'Institut d'Estudis Catalans, VII, 1921-1926, págs. 3-27

21 Antonio Beltran, Las pinturas esquemáticas de Lecina (Huesca), Zaragoza, 1972. A. Beltran y $\vee$. Baldellou, "Avance al estudio de las cuevas pintadas del barranco de Villacantal (Huesca)", Altamira Symposium, Madrid, 1980, págs. 131-140. V. BALDELLOU, "El arte rupestre postpaleolitico de la zona del rio Vero (Huesca)". Ars Praehistorica. III-IV, 19841985, págs. 111-137, 21 figs. (con la bibliografia anterior) 
Vero, es única en el arte prehistórico peninsular. En el extenso repertorio de sus representanciones se pueden encontrar muchos paralelos para el arte del valle del Segre, y utilizarlos hubiera estado más que justificado dada su cercanía geográfica. Una ojeada a los frisos pintados del Bajo Aragón tampoco hubiera estado de más.

Podriamos extendernos en algún otro detalle, pero no lo haremos pues se trata de cosas fácilmente subsanables para el lector advertido.

En resumen, lo que hay que proclamar es que el balance que se puede hacer de esta publicación es absolutamente positivo. El estudio del arte prehistórico, con sus continuos descubrimientos, se hace cada vez más complejo, muy en particular en los que se refiere a sus facies postpaleolíticas con los problemas de su sucesión estilística, de su cronologia, de su extraordinaria "comarcalización" y de su significado. Es muy de desear que pronto se pueda contar con otros volúmenes de este corpus arte rupestre de Cataluña, por ejemplo el de las ricas comarcas de ambas orillas del curso inferior del Ebro. Por todo ello queremos felicitar muy cordialmente a la "Diputació de Lleída" y a la "Direcció General del Patrimoni» del gobierno autónomo catalán por haber emprendido esta no fácil labor científica, inaugurando esta bella serie que tantos servicios rendirá a cuantos estudiamos el arte de nuestra Prehistoria. 